\title{
ON RELATED DIFFERENCE AND DIFFERENTIAL SYSTEMS†
}

BY W. M. WHYBURN

In a recently published article $\ddagger$ I considered the system of differential equations

$$
d y_{i} / d x=\sum_{j=1}^{j=m} A_{i j}(x) y_{j}+\beta_{i}(x), \quad(i=1, \cdots, m),
$$

where $A_{i j}(x), \beta_{i}(x),(i, j=1, \cdots, m)$ are summable, real functions of the real variable $x$ on $X: a \leqq x \leqq b$. I proved that for one set of definitions of the coefficients of the difference system

(2) $\Delta^{*} y_{i}(r) / \Delta x(r)=\sum_{j=1}^{j=m} A_{i j}(r) * y_{j}(r)+* \beta_{i}(r),(i=1, \cdots, m)$, on $E_{n}: x_{0 n}=a, x_{1 n}, \cdots, x_{n n}=b$, where the asterisk indicates a function defined on $E_{n}$ (replacing the bold-face type in my former paper), ${ }^{*} f(r)={ }^{*} f\left(x_{r n}\right)$ and $\Delta^{*} f(r)={ }^{*} f(r+1)-{ }^{*} f(r)$, every solution of this system goes over in the limit as $n$, the number of points in $E_{n}$, becomes infinite, in such a way that $X$ is completely subdivided to the corresponding solution, that is, the solution having the same initial values at $x=a$, of the differential system (1). The present paper shows that the conclusions of our former paper, stated above, are valid for all possible methods of defining the coefficients of system (2), so long as $\lim _{n \rightarrow \infty} * A_{i j}(p)=A_{i j}(x)$, $\lim _{n \rightarrow \infty} * \beta_{i}(p)=\beta_{i}(x)$, almost everywhere on $X$, and there exists a summable function $G(x)$ on $X$ such that $\left|{ }^{*} A_{i j}(p)\right|$, $\left|* \beta_{i}(p)\right|<G(x)$ for all $n,(i, j=1, \cdots, m)$, on $I_{p n}: x_{p n} \leqq x \leqq x_{p+1, n}$, where $p$ varies with $n$ in such a way that the point $x$ belongs to $I_{p n}$. It shows further that the approach to the limit is $u n i$ form on $X$ and that all of these conclusions are valid for any law of complete subdivision of $X$ by the points of $E_{n}$. Our former paper indicated ready adaptations of the work to non-

$\dagger$ Presented to the Society, November 29, 1929.

$\ddagger$ American Journal of Mathematics, vol. 51 (1929), pp. 265-286.

$\S$ By a complete subdivision is meant one such that $\lim n \rightarrow \infty$ maximum $\Delta x(i)=0$. 
linear systems and to systems containing parameters and the work of the present paper can be similarly adapted. Finally, an examination of the proofs together with the demonstrated uniformity of approach immediately yields estimates of the degree of the approximation of the solutions of the differential system by those of the difference system. The generality of the systems considered and the conclusions obtained cause the present paper to have an important bearing on several recent paperst and to contain some of the results of these papers as special cases.

Theorem. Let a method of complete subdivision of $X$ be given and let ${ }^{*} A_{i j}(r),{ }^{*} \beta_{i}(r),(i, j=1, \cdots, m ; r=0,1, \cdots, n)$, be defined for each $n$ so that $\lim _{n \rightarrow \infty} * A_{i j}(p)=A_{i j}(x), \lim _{n \rightarrow \infty} * \beta_{i}(p)=\beta_{i}(x)$, almost everywhere on $X$ and so that $\left|* A_{i j}(p)\right|,\left|* \beta_{i}(p)\right|<G(x)$ on $I_{p n},(p=0, \cdots, n-1)$, where $G(x)$ is summable on $X$. Let ${ }^{*} y_{i}(p),(i=1, \cdots, m)$, be any solution of $(2)$ and let $* y_{i}(0)$ $=\alpha_{i}$; then if $y_{1}(x), \cdots, y_{m}(x)$ is the solution of (1) such that $y_{i}(a)=\alpha_{i},(i=1, \cdots, m)$, we have $\lim _{n \rightarrow \infty} * y_{i}(p)=y_{i}(x)$ uniformly on $X$.

Proof. Examine \$1, pages 276-282, of my former paper. Using the notation of that paper, we establish two lemmas:

Lemma 1. $\operatorname{Lim}_{k \rightarrow \infty}{ }^{*} y_{k j}(p)=* y_{j}(p),(j=1, \cdots, m)$, uniformly with respect to $p$ and $n$.

LEMMA 2. $\operatorname{Lim}_{n \rightarrow \infty}{ }^{*} z_{k j}(p+1)=z_{k j}(x),(j=1, \cdots, m)$, uniformly on $X$.

Proof of Lemma 1. Let $*^{*} u(r)={ }^{*} g(r) \Delta x(r)$, where ${ }^{*} g(r)$ is the lower bound of $G(x)$ on $I_{r n}$ (existent since $G(x)>0$ ) and let $M=m\left[\alpha+\int_{a}^{b} G(x) d x\right]$, where $\alpha=\sum_{i=1}^{m}\left|\alpha_{i}\right|$. Repetition of the proof of page 278 of paper $\mathrm{J}$ shows that, for each $k$,

$$
\left|*_{z_{k j}}(p)\right|<\left(m^{k} M / k !\right)\left[\sum_{r=0}^{n-1} u(r)\right]^{k} \leqq D_{k},
$$

† See Plancherel, Bulletin des Sciences Mathématiques, (2), vol. 47 (1923), pp. 153-160, 170-177.

Bogoliouboff and Kryloff, Annals of Mathematics, (2), vol. 29 (1928), p. 255ff. Rose Whelan, this Bulletin, vol. 35 (1929), pp. 105-122.

$¥$ Whyburn, loc. cit. This paper will be referred to as paper J. It is to be noted that bold-faced letters in paper $\mathrm{J}$ are replaced by ordinary letters that are preceded by asterisks in the present paper. 
for all $n$ and $p$, where

$$
D_{k}=\left(m^{k} M / k !\right)\left[\int_{a}^{b} G(x) d x\right]^{k} .
$$

Since $D_{k}$ is independent of $n$ and $p$ and since the series of constants $\sum_{k=1}^{k=\infty} D_{k}$ is absolutely convergent, it follows that

$$
\lim _{k \rightarrow \infty}^{*} y_{k j}(p)=\lim _{k \rightarrow \infty} \sum_{r=0}^{k}{ }^{*} z_{r j}(p)={ }^{*} y_{j}(p)
$$

uniformly with respect to $p$ and $n$.

Proof of Lemma 2. We use mathematical induction. By the notation ${ }^{*} f(x)$, we mean a function that has the value $*_{f}(p)$ on $x_{p n} \leqq x<x_{p+1, n}$. (This notation replaces the previous notation $f^{(p)}(x)$ of paper J.) For $k=0$, we have

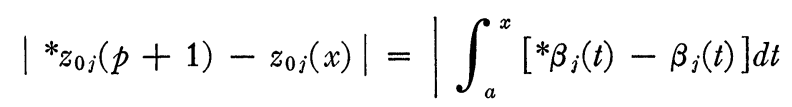

$$
+\int_{x}^{x_{p+1, n}} * \beta_{j}(t) d t\left|\leqq \int_{a}^{x}\right| * \beta_{j}(t)-\beta_{j}(t) \mid d t+\int_{x}^{x_{p+1, n}} G(t) d t
$$

for every $x$ on $X$. Let $\epsilon>0$ be assigned; then from the absolute continuity of $\int_{x}^{x} p+1, n G(t) d t$ and from the fact that the subdivision of $X$ is complete, we have that for every $n \geqq N_{1}$ and all $x$ on $X$, the last integral of (3) is less then $\epsilon / 2$. The limit as $n$ becomes infinite of the integrand in the first integral of (3) is zero almost everywhere on $X$ and this integrand is bounded by the summable function $2 G(x)$ for all $x$ on $X$ and for all $n$. An application of Lebesgue's theorem $\dagger$ shows that the limit as $n$ becomes infinite of this integral is zero. Hence for all $n>N_{2}$,

$$
\int_{a}^{b}\left|* \beta_{j}(t)-\beta_{j}(t)\right| d t<\epsilon / 2
$$

It follows from (3) that for all $n>N \geqq N_{1}, N_{2}$ and for all $x$ on $X,\left|* z_{0 j}(p+1)-z_{0 j}(x)\right|<\epsilon$. This establishes the lemma for $k=0$. We show that its validity for $k=r-1$ implies its validity for $k=r$. The expression $* z_{r j}(p+1)-z_{r j}(x)$ is made up of $m$ terms of which

$$
\sum_{i=0}^{p} * A_{j s}(i) z_{r-1, s}(i) \Delta x(i)-\int_{a}^{x} A_{j s}(t) z_{r-1, s}(t) d t
$$

is typical. Let $\epsilon>0$ be given; we show that each of the $m$ terms of the foregoing type is uniformly less than $\epsilon / m$ on $X$ for all $n>N$. Write such a term as $I_{1}-I_{2}+I_{3}$, where

$\dagger$ Leçons sur l'Intégration, etc., Paris, 1904, p. 114. 


$$
\begin{aligned}
I_{1} & =\int_{a}^{x} A_{j s}(t)\left[*_{z_{r-1, s}}(t)-z_{r-1, s}(t)\right] d t, \\
I_{2} & =\int_{a}^{x} *_{z_{r-1, s}}(t)\left[* A_{j s}(t)-A_{j s}(t)\right] d t, \\
I_{3} & =\int_{x}^{x_{p+1, n}}{ }^{*} A_{j s}(t) *_{z_{r-1, s}}(t) d t .
\end{aligned}
$$

From the validity of the lemma for $k=r-1$, we can choose $N_{1 s}$ such that for all $n>N_{1 s},\left|*_{z_{r-1, s}}(t)-z_{r-1, s}(t)\right|<\epsilon /\left[3 m \int_{a}{ }^{b} G(t) d t\right]$ on $X$. Hence for all $n>N_{1 s},\left|I_{1}\right|<\epsilon /(3 m)$ for every $x$ on $X$. Now for all $x$ on $X$,

$$
\left|I_{2}\right|<D_{r-1} \int_{a}^{b}\left|* A_{j s}(t)-A_{j s}(t)\right| d t
$$

and an application of Lebesgue's theorem $\dagger$ to the integral on the left-hand side of this inequality shows that for all $n>N_{2 s}$ this integral is less than $\epsilon /\left(3 m D_{r-1}\right)$ and hence $\left|I_{2}\right|$ is less than $\epsilon /(3 m)$ for every $x$ on $X$. From the absolute continuity of

$$
\int_{x}^{x_{p+1, n}} D_{k} G(t) d t
$$

and from the fact that the subdivision of $X$ is complete, we get $N_{3 s}$ such that for all $n>N_{3 s},\left|I_{3}\right|<\epsilon /(3 m)$ for every $x$ on $X$. Combining the results for $I_{1}, I_{2}, I_{3}$, and taking into account the $m$ terms that occur when $s=1,2, \cdots, m$, we get

$$
\left|*_{z_{r j}}(p+1)-z_{r j}(x)\right|<\epsilon, n>N \geqq N_{11}, N_{21}, N_{31}, N_{12}, \cdots, N_{3 m},
$$

and for every $x$ on $X$. This establishes Lemma 2 .

Proof of THEOREM. An argument $\ddagger$ entirely analogous to that used in proving Lemma 1 shows that $\lim _{k \rightarrow \infty} y_{k j}(x)=y_{j}(x)$ uniformly on $X$. Hence if $\epsilon$ is an assigned positive number there exists $K_{1}$ so that for $k>K_{1},\left|y_{k j}(x)-y_{j}(x)\right|<\epsilon / 3$ on $X$. In accordance with Lemma 1 , let $K$ be chosen greater than $K_{1}$ and so that for all $n$ and $p$,

$$
\begin{aligned}
& \left|{ }^{*} y_{K}(p)-*^{*} y_{j}(p)\right|<\epsilon / 3, \\
& \left|y_{K}(x)-y_{j}(x)\right|<\epsilon / 3, \quad \text { for all } n \text { and } p,
\end{aligned}
$$

$\dagger$ Loc. cit.

$\ddagger$ This is but the ordinary argument for successive approximations. 
By Lemma 2 choose $N$ so that for all $n>N$,

$$
\left|*_{z_{r j}}(p)-z_{r j}(x)\right|<\frac{\epsilon}{3(K+1)}, \quad(r=0, \cdots, K),
$$

and all $x$ on $X$. From

$$
*_{y_{K} j}(p)=\sum_{r=0}^{K} *_{z_{r j}}(p), \quad y_{K j}(x)=\sum_{r=0}^{K} z_{r j}(x),
$$

and (6), follows

(7) $\left|*^{*} y_{K}(p)-y_{K j}(x)\right|<\epsilon / 3$, for $n>N$ and all $x$ on $X$.

A combination of (4), (5), and (7) yields

$$
\left|y_{j}(x)-* y_{j}(p)\right|<\epsilon, \quad \text { for all } n>N \text { and for every } x \text { on } X \text {. }
$$

This establishes the desired uniformity and completes the proof of the theorem.

Obviously one need but check through the foregoing work and that of paper $\mathrm{J}$ to obtain estimates of the degree of approximation. $\dagger$

The University of California at Los Angeles

$\dagger$ The approximations obtained in this way can of course be improved when more special systems are considered. 